\title{
Analysis of Energy Efficiency using Improved MAC in Wireless Sensor Network Environments
}

\author{
Cheol-seung Lee \\ Dept. of Teacher Training \& Liberal Arts, \\ Kwangju Women's University, Gwangju, Korea \\ cyberec@kwu.ac.kr
}

\begin{abstract}
In this paper, we designed and analyzed a MAC protocol that is adaptive to traffic and improved energy efficiency in a WSN(wireless sensor network) environment.

Due to the development of ubiquitous computing environment, Wireless sensor network technology is being applied in various fields and fusion research is being underway. The wireless sensor network allows wireless sensor nodes to communicate with each other in a distributed network environment and collects data of the necessary area as a connected structure also, it is a structure that connects collected data to a wired network through a sink. First of all, it is very important to manage the energy more efficiently than the QoS requirement because the wireless sensor nodes maintain the life cycle using the battery. In consideration of these issues, the IEEE 802.15.4 MAC protocol is studying traffic adaptive MAC protocol and standardization work emphasizing reliability and efficiency in a wireless sensor network environment. However, the response speed of the sensor node is lowered as the energy efficiency of the wireless sensor node is reduced.

Therefore, in this paper, we analyze the MAC protocol of synchronous and hybrid methods, and design the MAC protocol with improved energy efficiency of the whole network and the safety and the efficiency are mathematically proved and analyzed through experiments.
\end{abstract}

Keywords: Wireless Sensor Network, MAC Protocols

\section{Introduction}

In the Ubiquitous computing environment, the wireless sensor network technology is establishing a new paradigm by sharing information between objects as well as people and objects through various forms of application such as traffic monitoring and tracking technology, military purpose, mobile device detection, health monitoring and smart environment[1][2].

As shown in Figure 1, a wireless sensor network is a structure in which many wireless sensor nodes communicate with each other collects data of a necessary area and connects the collected data to a wired network through a sink.

Due to the development of hardware technology, the development of sensor nodes has been able to collect information by distributing sensor nodes equipped with various sensing modules to a wide area, studied in various ways[3]. However, since sensor nodes use energy that can operate for a certain period of time, that is, the battery, it is very important to maximize the efficiency of the battery[4]. Sensor nodes must work until the battery is exhausted, It is restricted by energy use. If the source node's battery is exhausted, it can no longer join the network, so energy consumption should be considered as much as possible[5][6].

Received (April 5, 2017), Review Result (July 21, 2017), Accepted (September 15, 2017) 


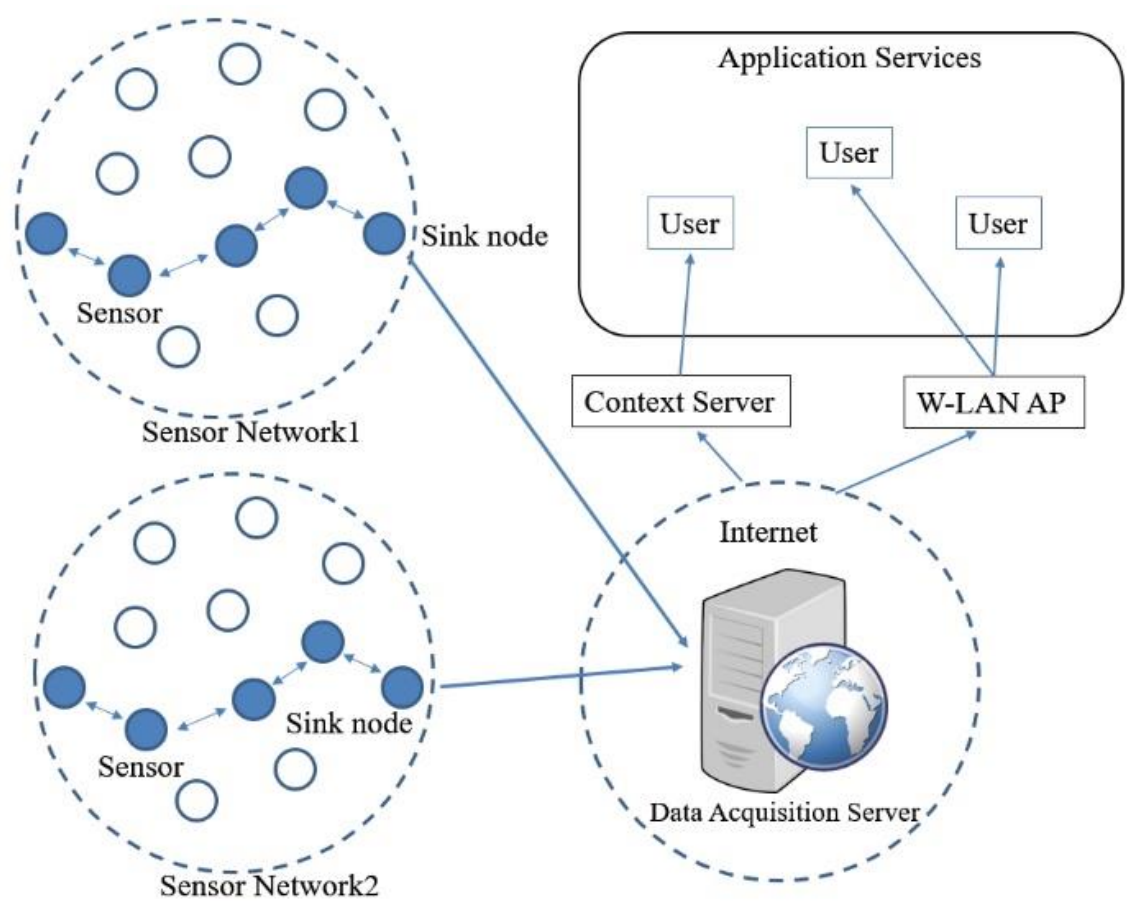

Figure 1. Wireless Sensor Network Environment

Although the reliability of the wireless sensor network environment and the standardization work of the information transmission of the sensor node are supplemented by the function of the MAC standard of IEEE802.15.4 in the TG4e, In order to minimize the power loss, it is necessary to study how to reduce the data transmission speed and response speed due to the problem of the data transmission speed in the sleep mode of the sensor node and the response speed as the power consumption is reduced.

In order to improve the efficient MAC protocol, this paper proposes a protocol that can receive beacon dynamically according to the data transmission frequency, thereby minimizing the efficient power management and response speed.

\section{Related Research}

\subsection{Standardization of MAC Technology of Wireless Sensor Network}

In order to overcome MAC standard technology, Maintain compatibility, and Functional limit, IEEE802.15 TG4e of MAC technology of wireless sensor network has all of a plurality of actions in many service areas such as IEC 62591, ISA100.11a, WIA-PA(Wireless Network for Industrial Automation Process Automation). The sensor network technology such as DSME(Distributed Synchronous Multichannel Extension) mode, TSCH(Time Slotted Channel Hoping) mode, LL(Low Latency) mode and RFID BLINK frame support frame support mode is applied so that the user can select the MAC mode according to the purpose and operate the network, thereby contributing to the active WPAN market.

The operation of IEEE802.15TG4e-based PAN is divided into beacon mode in which PAN is operated based on beacon periodically broadcasted and non-beacon mode in which non-periodic beacon is requested for communication frame exchange. Figure 2 illustrates the operation of IEEE802.15TG4e-based PAN. 


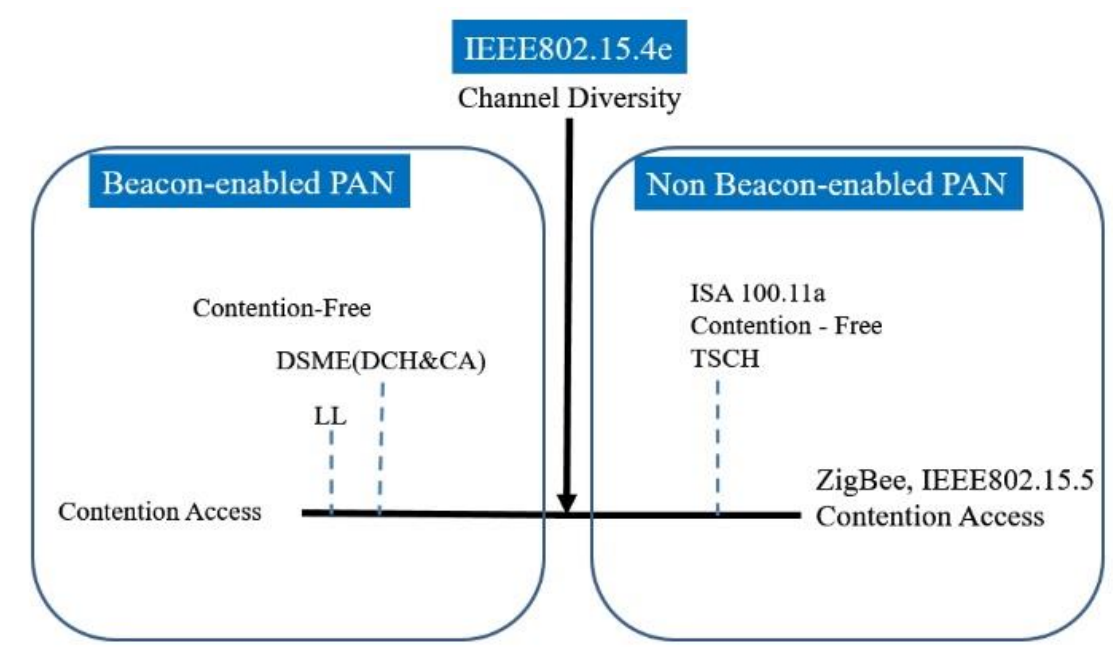

Figure 2. IEEE 802.15.4e MAC Operation Mode

\subsection{MAC Protocol}

IEE 802.15 wireless sensor network MAC protocol standardization technology TG5 is a group that establishes a MAC protocol standard for low-cost products with lower data rates and battery of source nodes lasting several years through WPAN Mesh network type MAC and PHY studies[7].

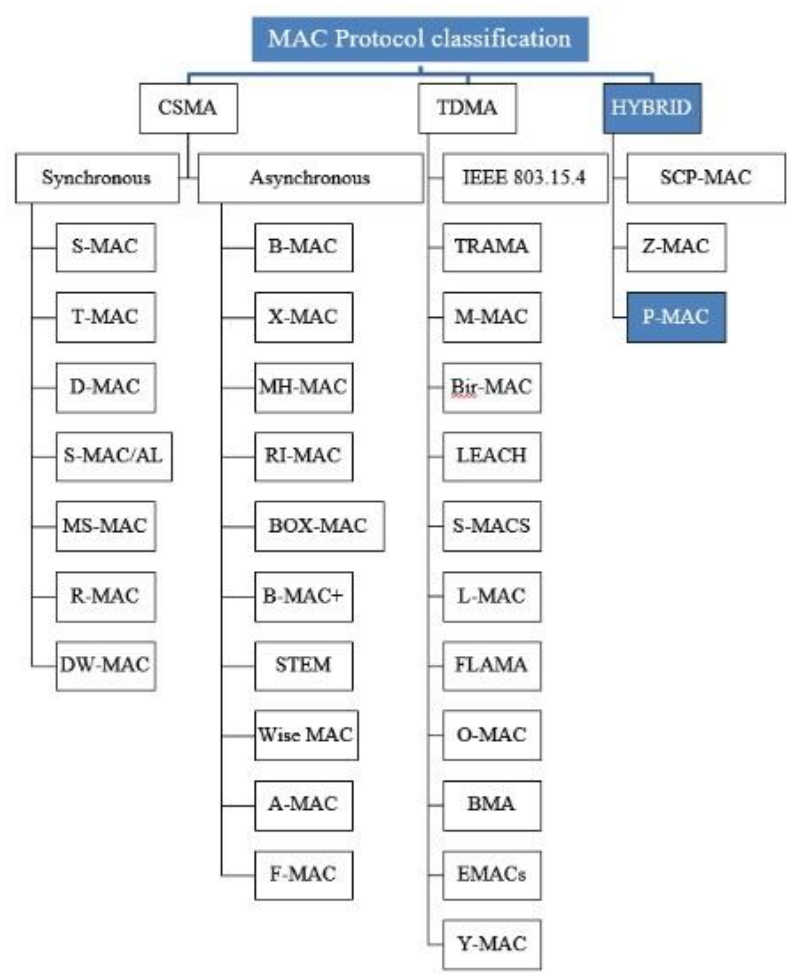

Figure 3. MAC Protocol Classification

In a wireless sensor network, unlike a general wireless network, it can be divided into data transmission / reception, idle listening, and sleep mode consumes the most energy when transmitting data. The idle listening state is waiting when the power is maintained to receive data of the neighboring sensor node, and consumes little energy in the sleep mode. 
In wireless sensor network, MAC protocol is proposed to minimize energy consumption rather than QoS requirement because it operates based on battery. Figure 3 lists the MAC protocols used in wireless sensor networks according to classification. The research on Sensor MAC(S-MAC), Timeout MAC(T-MAC), and Pattern MAC(P-MAC) considering characteristics of sensor nodes in wireless sensor networks is typical.

\subsubsection{Sensor MAC}

The Sensor MAC protocol has been proposed to solve the unnecessary idle listening problem of the wireless sensor network by periodically repeating the active mode and the sleep mode. The active mode and the sleep mode, which are the active period and the inactive period, Is a frame periodically constructed with a predetermined length [8][9].

During the sleep mode, the power of the transceiver related to the sensing is turned off, so that the data is not transmitted but stored in the buffer, thereby extending the entire network lifetime. In the active mode, it checks whether transmission / reception is performed according to the presence of data stored in the buffer, and transmits data to neighboring sensor nodes.

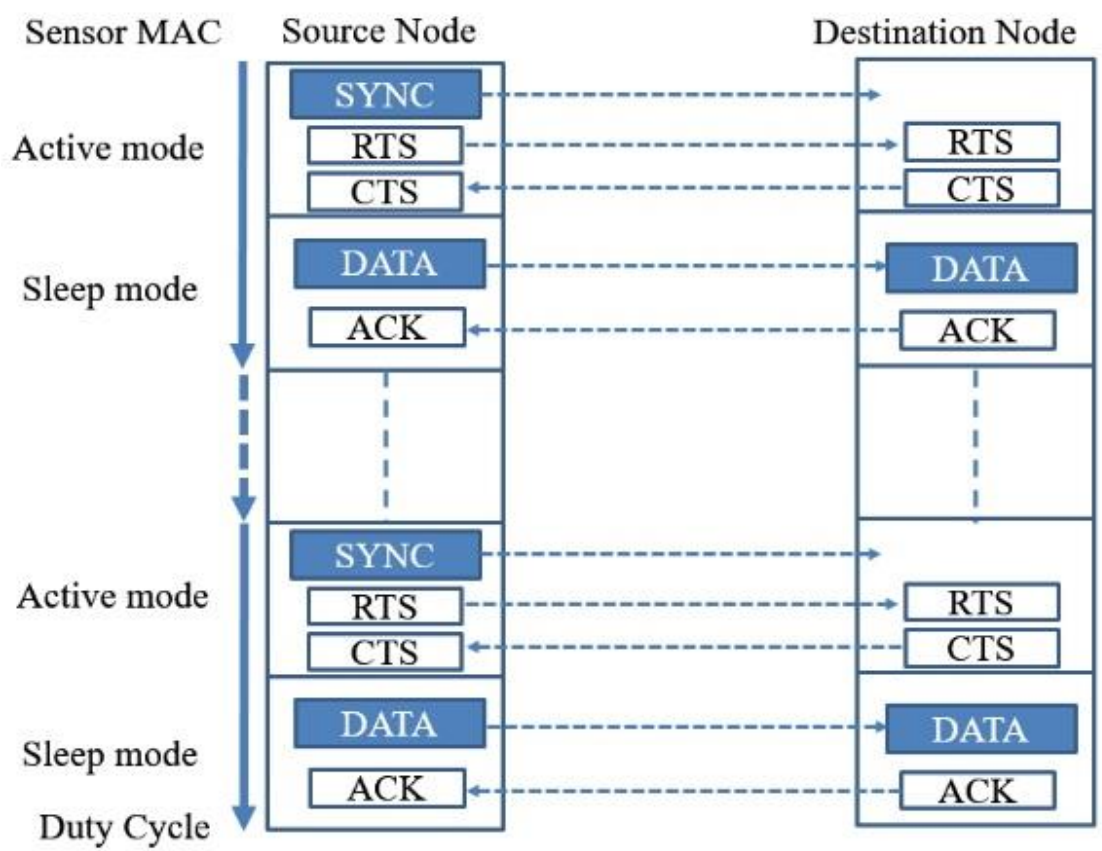

Figure 4. Sensor MAC Protocol Operation

Figure 4 shows the operating status of the Sensor MAC protocol. the SYNC part is for synchronization among neighboring sensor nodes using SYNC packets, and the DATA part is for data transmission using the RTS(Request To Send) / CTS(Clear To Send) handshake mechanism as in 802.11.

After sending SYNC control packet in active mode of source node, it sends RTS packet. The destination node transmits a CTS packet in response to the RTS packet, and the data transmission can confirm whether the received data exists or not during the sleep mode. The data transmission speed is reduced during the sleep mode, and the data transmission is efficient due to the simple implementation. However, the duty cycle is fixed based on the high load, which is disadvantageous in an environment in which traffic changes. 


\subsubsection{Timeout MAC}

The Timeout MAC[10] protocol, which improves the problem of the duty cycle of the Sensor MAC protocol, exchanges RTS packets and CTS packets of protocol sensor nodes using a synchronous timer and transmits data packets using ACK(Acknowledge) and reliability is ensured. The Timeout MAC protocol operates in the same synchronization mode as the Sensor MAC protocol, but if data transmission / reception is not detected in the active mode, It can increase efficiency by reducing the energy consumed by idle listening.

Figure 5 shows a Timeout MAC using a synchronous timer. The Timeout MAC uses a timer of a fixed time, Tn, by using the length of the contention period, the length of the RTS packet, and the return time. If there is no data reception during the Tn time, the receiving node switches to the sleep mode in the inactive section, It is a MAC protocol that improves efficiency by reducing unnecessary idle listening energy consumption.

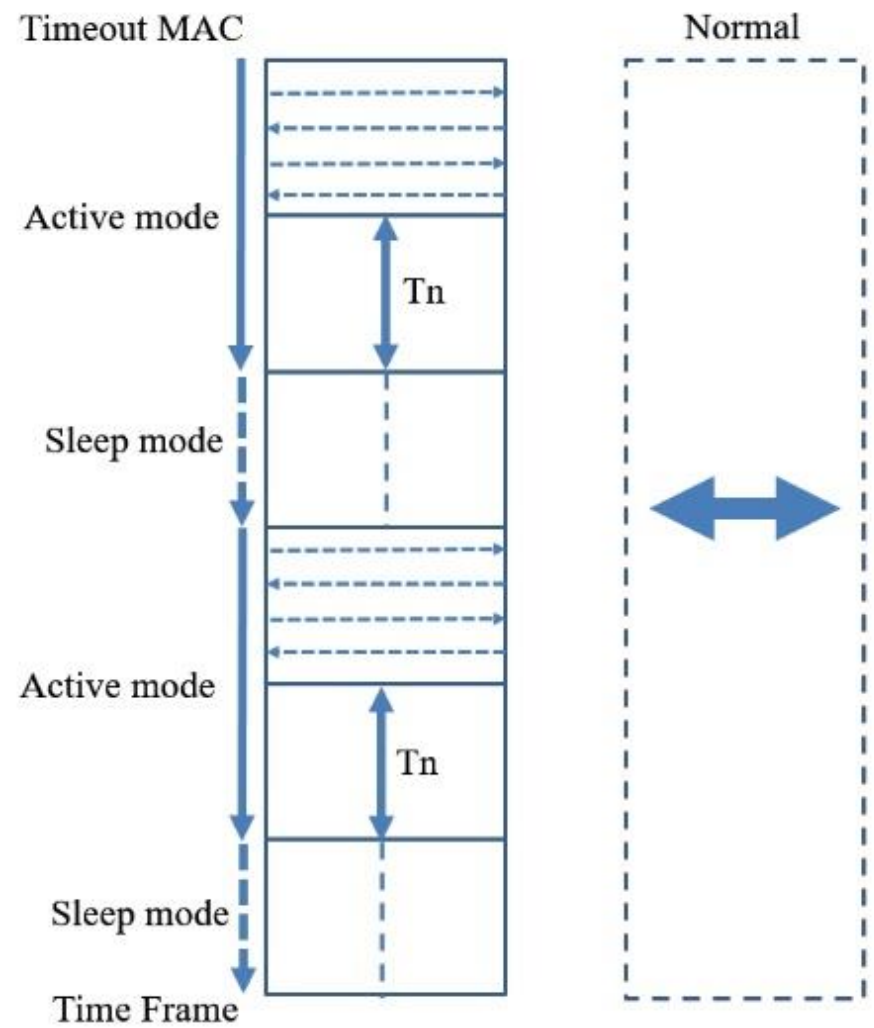

Figure 5. Timeout MAC Protocol Operation

\subsubsection{Pattern MAC}

In the Pattern MAC [11] protocol, a segmented time section is applied as in the Sensor MAC protocol, but the pattern exchange is performed according to the dynamic traffic in which the data transmission amount of the sensor nodes participating in the network changes, each sensor node dynamically schedules active mode and sleep mode based on the traffic patterns of neighboring nodes. 


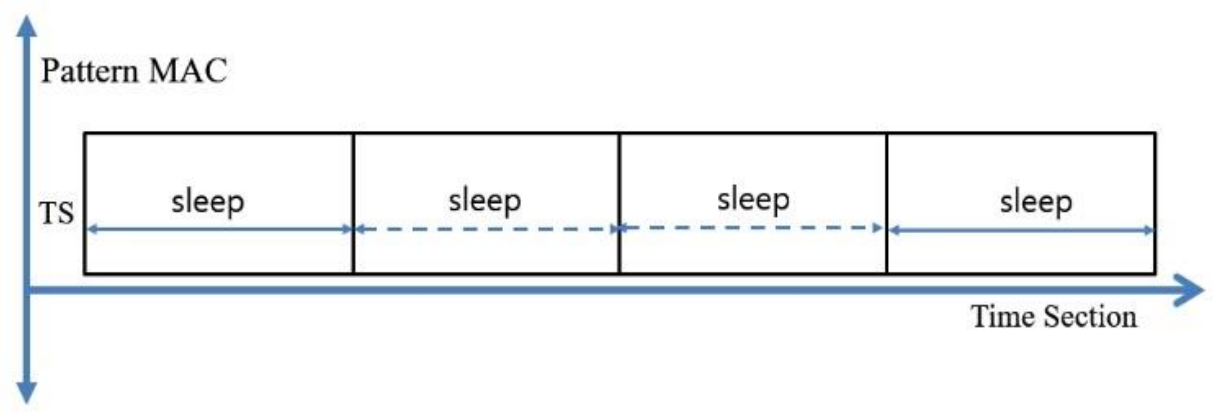

Figure 6. Pattern MAC Protocol Operation

The P-MAC protocol is divided into STFs(Super Time Frames) and consists of two PETF(Pattern Exchange Time Frame) and PRTF(Pattern Repeat Time Frame). In the PETF, sensor nodes collect their repeating traffic patterns with neighboring sensor nodes, find the pattern type of the data sent previously through exchanges, schedule in the active mode of the next interval according to the current pattern. It can manage energy efficiently by reducing traffic load.

\subsection{Problems of MAC Protocol in Chapter 2}

In wireless sensor networks, MAC protocols have been studied to reduce energy consumption during idle listening of sensor nodes. Although protocols such as Sensor MAC, Timeout MAC and Pattern MAC are representative of energy-efficient protocols, there are still many problems.

In case of Sensor MAC, collision may occur due to hidden terminal problem when data is transmitted from two or more transmitting nodes in a multi-hop state, and unnecessary energy is consumed as fixed wakeup when data transmission does not occur, and transmission delay may occur in fixed sleep mode.

In the case of Timeout MAC with improved Sensor MAC protocol, it is difficult to synchronize the data transmission of all sensor nodes as the network size increases. Also, there is a disadvantage that it is difficult to accurately obtain the length of the contention period, the length of the RTS packet, and the return time for setting the Tn time.

The time-slotted structure of Pattern MAC requires high time synchronization that requires high network maintenance overhead, and has a problem of long transmission delay and low network efficiency.

The MAC protocol needs to improve these problems and requires research and proposals focused on energy efficiency rather than QoS requirements. Chapter 3 of this paper proposes energy efficiency studies.

\section{Energy Efficiency Proposal Study}

The MAC protocols of wireless sensor networks have been studied in such a way that the sensor nodes reduce the power consumption by minimizing the active mode time, However it has the disadvantage that the response speed drops as the power consumption decreases.

In this section, we propose a dynamic sleep protocol that schedules the sleep mode of a sensor node by configuring a wireless sensor network based on the Pattern MAC protocol. The information of the packet data of the sensor node includes the received RSSI value of the node to be transmitted and the received transmission power. The sensor node receiving the packet data can know the RSSI value of the actual transmitting node and how far away from the sensor node the packet data is through the set value to receive the received RSSI value. Based on 
this, it is possible to set the appropriate transmission power when sending the response message, so that the power consumption of the sensor node can be reduced a little.

However, the RSSI value causes errors due to environmental effects Even if the measured value is the same as the experiment, the result may be different, and it only plays a role of reducing the power consumption of the sensor node [12].

\subsection{Pattern Application Scheduling}

For energy efficiency research, the sensor node analyzes the pattern of previously received data, and then studies the dynamic sleeping protocol that schedules the sleep mode based on this pattern.

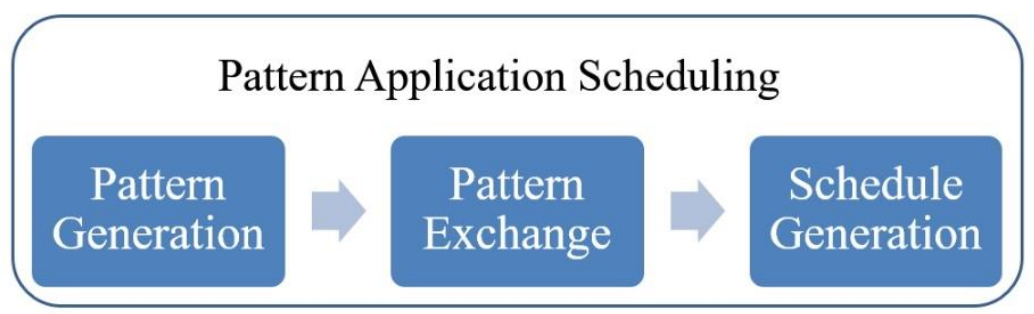

Figure 7. Pattern Application Scheduling

The pattern is generated by applying its own traffic and neighboring nodes' sleep mode and active mode. the time section to which a pattern is applied consists of $\mathrm{n}$ equal time sections and is expressed as TS (Time Section) $=\left\{P_{0}, P_{1}, P_{2}, P_{3}, \ldots \ldots \ldots . P_{n-1}, P_{n}\right\}$.

The generated pattern is represented by $P_{i, n}^{j}$, and the time section to which the $i$-th period and the $n$-th pattern of the sensor node $\mathrm{j}$ are applied is composed of $\mathrm{n}$ time section equal to each other.

$$
T S=\left\{P_{1,0}^{j}, P_{1,1}^{j}, P_{1,2}^{j}, P_{1,3}^{j}, \ldots \ldots \ldots \ldots . P_{1, n-1}^{j}, P_{1, n}^{j},\right\}-----\quad \text {--- (Equation 1) }
$$

The interval in time section at which the pattern is applied in Equation 1 must be set to active mode, and the sleep mode can be set up to the maximum interval. TS is determined according to the amount of data collected in the TS-1 time section, and is applied from the beginning of the pattern, and the pattern once set is maintained until the interval of the TS+1 time section.

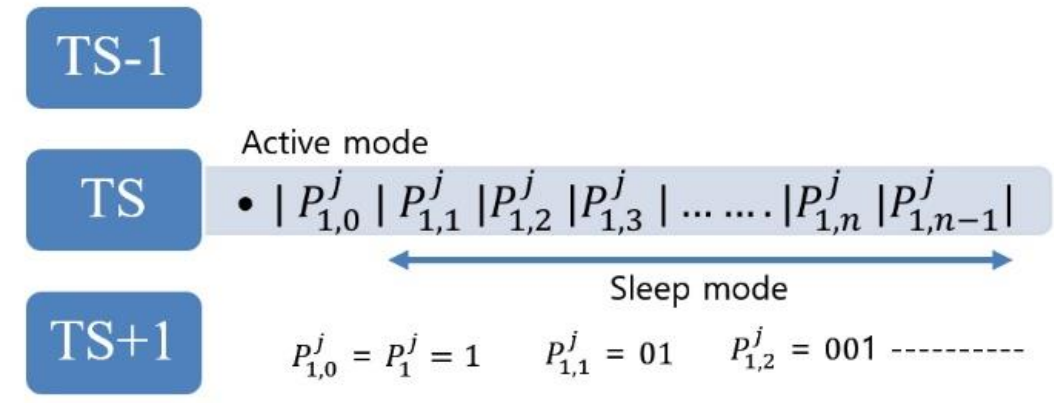

\section{Figure 8. Time Section Scheduling}

The TS +1 th time section is determined according to the pattern from $P_{1,0}^{j}$ to $P_{1, n}^{j}$ in TS section, and the section from $P_{1,0}^{j}$ to $P_{1, n}^{j}$ in TS section is active mode, and the pattern to be applied to the next section is updated. if the amount of change in the data of the Ts-1 period is less or equal, the sensing period is increased by applying the sleep mode. 
$\left(\left\{1_{0}, 1_{1}, 1_{2}, 1_{3}, \ldots \ldots 1_{n-1}, 1_{n}\right\} \rightarrow\left\{1_{0}, 0_{1}, 1_{2}, 0_{3}, \ldots \ldots 1_{n-1}, 0_{n}\right\}\right)$ - (Equation 2)

If the amount of change of the data in the TS-1th section is small even in the TS + 1th time section, the sleep mode is increased by $2 \mathrm{~K}$. When the amount of change of data in the TS-1 period is increased, the sleep mode interval is decreased to increase the sensing period for the interval where the data change amount is large, thereby improving the network response speed and data reliability.

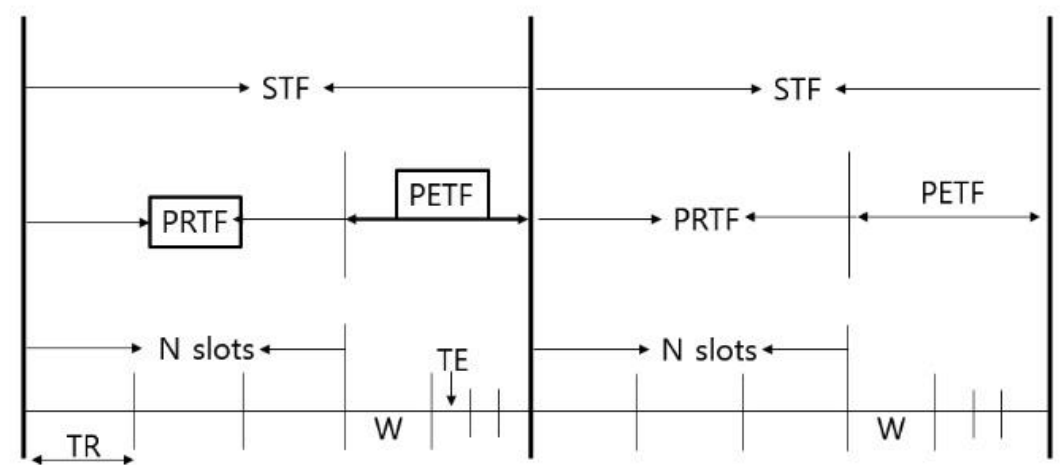

STF(Super Time Frames)

PRTF(Pattern Repeat Time Frame)

PETF(Pattern Exchange Time Frame)

$\mathrm{N}$ slots : The maximum number of neighbor nodes a node can have

$\mathrm{TR}=\mathrm{CW}+\mathrm{RTS}+\mathrm{CTS}+\mathrm{DATA}+\mathrm{ACK}$

$\mathrm{TE}=$ long enough to broadcast a pattern

$\mathrm{N}$ increase $=$ Energy decrease, Latency increase

Figure 9. Division of Time Frames

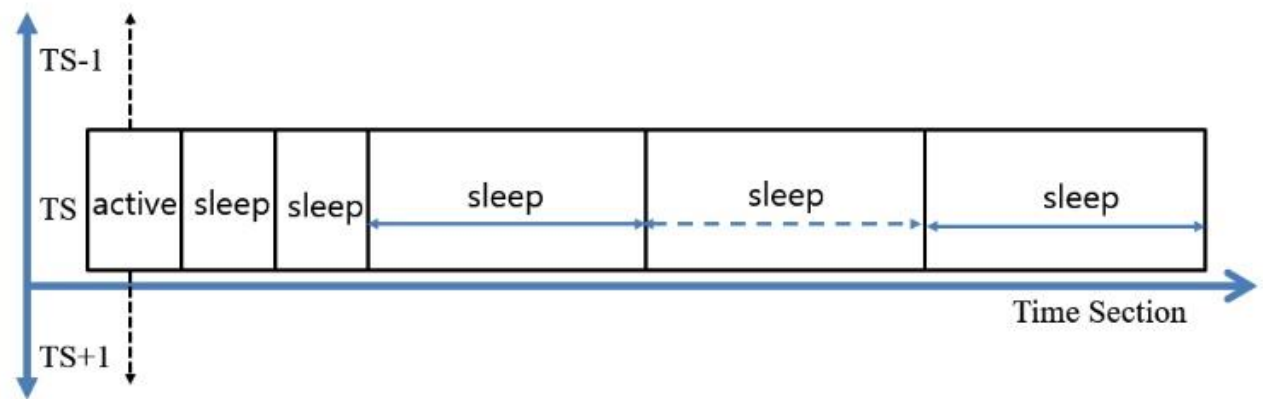

Figure 10. Pattern Application and Schedule

\subsection{Threshold( $(\delta)$ Setting based on Data Variation}

The measured data value at the sensor node of the wireless sensor network may have an difference value $\delta$. The sleep mode is shortened according to the value, which increases the energy consumption of the sensor node. In order to measure the data change efficiently, it is necessary to set the difference range. The value varies depending on the sensitivity of the sensor and may be slightly different depending on the surrounding environment (temperature, humidity), but it is usually a constant value.

If the threshold is set low, the sensor's sleep mode is shortened and the network continues to operate. Therefore, depending on the data or environment to be applied, it is necessary to allow an error of a proper value and decide on the situation to be used.

If there is no data for node $\mathrm{j}$ to send during period 1 , the following sequence of patterns is generated at node $\mathrm{j}$. 


$$
\begin{gathered}
1,01,0^{2} 1,0^{4}, \ldots \ldots \ldots \ldots \ldots 0^{\delta} 1,0^{\delta} 01 \\
0^{\delta} 0^{2} 1,0^{\delta} 0^{3} 1, \ldots \ldots \ldots \ldots \ldots 0^{n-1} 1 .
\end{gathered}
$$

\section{Performance Evaluation}

The proposed energy efficiency study measured the energy efficiency using the patterns and $\delta$ values of the sensor nodes.

Table 1. Simulation Environment

\begin{tabular}{|l|l|}
\hline Configuration & Model \\
\hline CPU & Intel i7-6700HQ 2.6GHz \\
\hline RAM & $16 \mathrm{~GB}$ \\
\hline Network Simulator & NS3 \\
\hline Language & C, C++ \\
\hline Sensor node & 50 nodes \\
\hline Transport & UDP \\
\hline Simulation Time & $0 \sim 1500 \mathrm{sec}$ \\
\hline TR & $258 \mathrm{~ms}$ \\
\hline TE & $104 \mathrm{~ms}$ \\
\hline PRTF & 64 slots \\
\hline PETF & 4 slots \\
\hline
\end{tabular}

For the experiment of the proposed algorithm, NS3 was used and the simulation time was set to 1500 seconds, and 50 arbitrary sensor nodes were selected and compared with the Sensor MAC protocol and Pattern MAC protocol to measure the energy efficiency.

$$
\text { Energy efficiency }=\frac{\text { Total throughpht }}{\text { Total energy consumption }}
$$

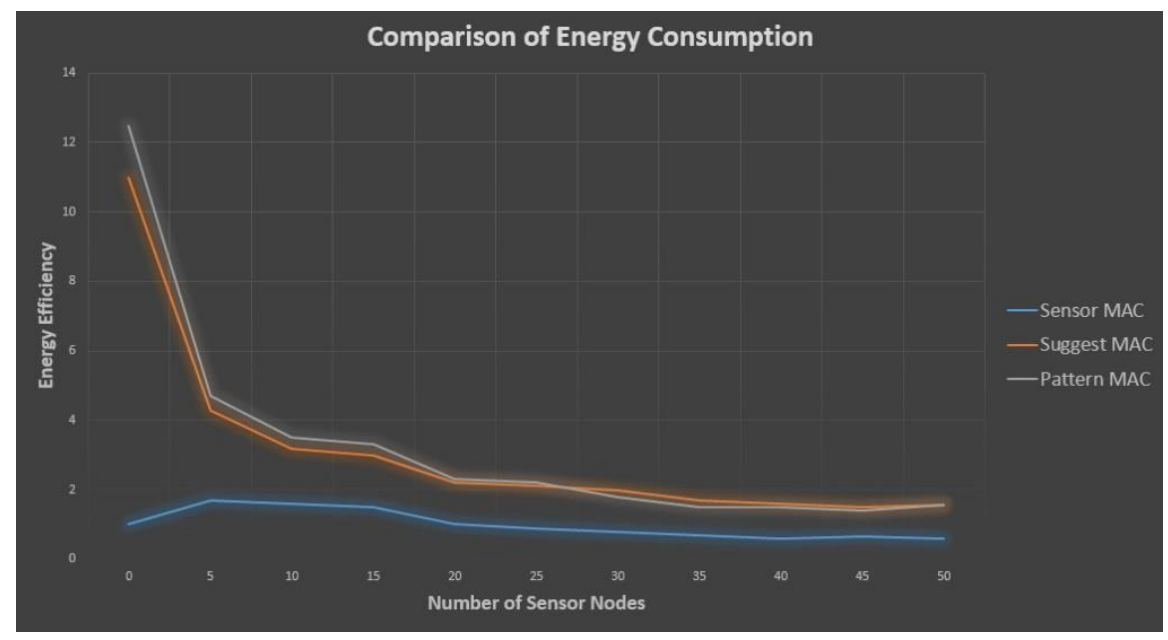

Figure 11. Energy Efficiency Measurement

Figure 11 shows the energy efficiency of Sensor MAC protocol, Pattern MAC protocol and suggested MAC protocol using 50 arbitrary sensor nodes.

At the beginning of the experiment, the suggested protocol showed better energy efficiency than the Sensor MAC in the sleep mode. As the number of participating sensor nodes in the whole network increases, As a result, the energy consumption is 
increased and the overall energy efficiency is lowered. However, when compared to the Sensor MAC protocol, the suggested protocol based on Pattern MAC is energy efficient even if the number of sensor nodes increases. In addition, the suggested MAC protocol shows energy efficiency similar to that of the pattern MAC even if the number of nodes increases, which is efficient.

\section{Conclusion and Future Research}

The development of wireless sensor network technology has been applied and studied in various fields of Ubiquitous computing. Sensor nodes participating in a wireless sensor network must last a long period of life through efficient energy consumption for a long time, It consumes a lot of energy to search neighboring sensor nodes or receive data.

First of all, it is very important to manage the energy more efficiently than the QoS requirement because the wireless sensor nodes maintain the life cycle using the battery. In consideration of these issues, the IEEE 802.15.4 MAC protocol is studying traffic adaptive MAC protocol and standardization work emphasizing reliability and efficiency in a wireless sensor network environment. However, the response speed of the sensor node is lowered as the energy efficiency of the wireless sensor node is reduced.

In this study, we designed a wireless sensor network to enable active mode and sleep mode scheduling of sensor nodes by applying hybrid P-MAC protocol. The sensor node compares the sleep mode with previously collected sensor data, By allocating the sleep mode dynamically and including the distance information through the RSSI value in the packet data information, This study is to increase the energy efficiency of sensor nodes participating in the entire network.

Considering the growth potential of the wireless sensor network that will be the foundation of the fourth industrial revolution era, it should be lightly designed so that the battery duration can be maintained for a long time considering the calculation amount of the sensor nodes. In addition, a secure communication path among the sensor node and sink node and Acquisition Server must be secured, It is necessary to provide a robust wireless sensor network environment by applying the security technique with enhanced reliability and efficiency between the nodes. The development and continuous research of convergence technology of wireless sensor network is expected to be put to practical use in various industrial fields.

\section{Acknowledgments}

This paper is a revised and expanded version of a paper entitled "A Study on Low Power using Improved MAC Protocol based on WSN" presented at, C.-S. Lee, Ho Chi Minh, Vietnam, August 16-18 of Proceedings International Conferences NGCIT 2017 and ISI 2017.

This paper was supported(in part) by Research Funds of Kwangju Women's University in 2017 (kwul17-049).

\section{References}

[1] P. Schaffer, K. Farkas, A. Horvath, T. Holczer and L. Buttyan, "Secure and Reliable Clustering in Wireless Sensor Networks: A Critical Survey”, Computer Networks, vol. 56, no. 11, (2012), pp. 27262741.

[2] W. Heinzelman, A. P. Chandrakasan and H. Balakrishnan, "An Application-Specific Protocol Architecture for Wireless Microsensor Networks", IEEE Trans. Wireless Communications, vol. 1, no. 4, (2002), pp. 660-670.

[3] P. Hung, L. Xiao, S. Soltani, M. Mutka and N. Xi, "The evolution of MAC protocols in wireless sensor networks: A survey", J. of The IEEE Communications Surveys \& Tutorials, vol. 15, Issue 1, (2012), pp. 101-120. 
[4] W. Oh and S. Lee, "MAC protocol for Energy-Efficiency and Delay in Ubiquitous Sensor Networks", J. of the Korea Institute of Electronic Communication Sciences, vol. 4, no. 1, (2009), pp. 19-23.

[5] J. Lee and I. Kim, "Energy-efficient Channel Allocation MAC for Wearable WBANs", J. of the Korea Institute of Electronic Communication Sciences, vol. 11, no. 11, (2016), pp. 1135-1140.

[6] Y. Jo, I. Yun, S. Kim and H. Park, "A Study on the MAC(Media Access Control) protocol for Unmanned Aerial Vehicle(UAV)", J. of the Korea Institute of Electronic Communication Sciences, vol. 11, no. 1, (2016), pp. 119-124.

[7] Standard 802.15.4-2006, Part 15.4: Wireless Medicum Access Control (MAC) and Physical Layer (PHY) Specifications for Low-Rate Wireless Personal Area Networks (WPANs), New York, USA, (2006).

[8] W. Ye, J. Heidemann and D. Estrin, "Medium Access Control with coordinated Adaptive Sleeping for Wireless Sensor Networks", J. of the IEEE/ACM Trans. Networking(TON), vol. 12, issue 3, (2004), pp. 493-506.

[9] W. Ye, J. Heidemann, and D. Estrin, "An Energy-Efficient MAC Protocol for Wireless Sensor Network", In Proc. the $21^{\text {st }}$ Annual Joint Conf. of the IEEE Computer and Communications Societies (INFOCOM 2002), vol. 3, New York, USA, (2002).

[10] T. Dam and K. Langendoen, "An Adaptive Energy-Efficient MAC Protocol for Wireless Sensor Networks," In Proc. the $1^{\text {st }}$ Int. Conf. on Embedded Networked Sensor System (SenSys'03), Los Angeles, USA, (2003), pp. 171-180.

[11] T. Zheng, S. Radhakrishnan and V. Sarangan, "PMAC: An Adaptive Energy Efficient MAC Protocol for Wireless Sensor Network", In Proc. the $19^{\text {th }}$ IEEE Int. Parallel and Distributed Processing System, Denver, USA, (2005).

[12] C. Lee, "A Study on Low Power using Improved MAC Protocol based on WSN", In Proc. the $6^{\text {th }}$ Int. Conf. on Next Generation Computer and Information Technology (NGCIT 2017), Ho Chi Min, Vietnam, (2017).

\section{Author}

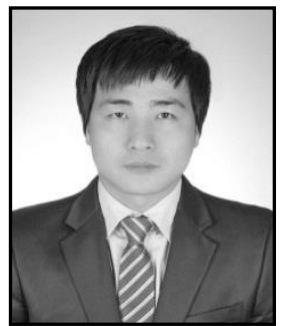

Cheol-seung Lee, he is currently an assistant professor at the Teacher Training \& Liberal Arts Department at the University of Kwangju women's University in Korea. He received his Ph.D. in Computer Engineering from the University of Chosun, Korea, in 2008.

His recent research activites are focused on Mobile Ad-hoc Network security and Mobile programming and privacy in IOT and smart environments. 
International Journal of Control and Automation

Vol. 10, No. 11 (2017) 